\title{
Acercamiento al Campo de la Psicología Aeronáutica $^{1}$
}

\section{Approach to the field of Aeronautical Psychology}

\author{
Felipe Galeano Arias ${ }^{2}$
}

\section{Recibido: 2018-12-11 Aceptado: 2019-03-22}

Resumen: El impacto de la psicología en el sector aeronáutico es innegable. Este artículo presenta un estudio de profundización que indaga la forma en la cual el sector aéreo ha recurrido a la ciencia que indaga por las motivaciones del comportamiento, y trata de que ésta permita entender de qué manera la actuación humana se ajusta con los objetivos que buscan fomentar y mantener la seguridad operacional a través del estudio e intervención sobre el elemento humano y la comprensión de los Factores Humanos en Aviación. Se hace un recorrido por la definición del campo y la forma en que éste, desde sus comienzos, tuvo un interés por las respuestas comportamentales humanas en las condiciones de vuelo, así como preguntas sobre la selección de las personas que intervienen en la operación aérea y finalmente, el impacto de este campo en el contexto colombiano. El presente texto pretende situar cómo se ha definido la psicología aeronáutica, cuál es su objeto de estudio y la concepción de sujeto al interior de esta. Así mismo, plantear algunas de las limitaciones del hacer del psicólogo en este campo.

Palabras clave: psicología aeronáutica, factores humanos en aviación, campos de la psicología, actuación humana, historia de la psicología.

Abstract: The impact of psychology in the aeronautical sector is undeniable. This article presents an in-depth study that investigates the way in which the air sector has resorted to science that investigates behavioral motivations, and tries to make it possible to understand how Human Performance fits with the objectives they seek. promote and maintain safety through the study and intervention on the human element and the understanding of Human Factors in Aviation. It takes a tour of the definition of the field and the way in which it, from its beginnings, had an interest in human behavioral responses in flight conditions, as well as questions about the
Para citar este artículo en APA: Galeano, F. (2019). Acercamiento al campo de la Psicología Aeronáutica. Revista de Psicología Universidad de Antioquia, 11(1), 195-218. DOI: https://doi.org/10.17533/udea. rp.v11n1a09
Investigación realizada en el marco de la Especialización en Psicopatología y Estructuras clínicas, Universidad de Antioquia, Medellín, Colombia.

2 Psicólogo, Máster en Dirección y Administración de Recursos Humanos, Especialista en Psicopatología y Estructuras clínicas. Actualmente se desempeña como Coordinador Gestión Competencias Avanzadas en Avianca. Correo: felipe.galeano@avianca.com; https://orcid.org/0000-0001-7812-700X 
selection of people involved in the air operation and finally, the impact of this field in the Colombian context. The present text tries to locate how the aeronautical psychology has been defined, which is its object of study and conception of the subject within the same.
Also, raise some of the limitations of the psychologist's work in this field.

Keywords: Aeronautical psychology, human factors in aviation, fields of psychology, human performance, History of Psychology.

\section{Contextualización epistemológica}

El desarrollo de la psicología aeronáutica ha estado ligada al desarrollo de las aeronaves como medio de transporte, así como máquinas de guerra; que tal como lo afirma Alonso (2012) es el más seguro que existe para el ser humano. De ahí que este sea considerado como "la parte más flexible, adaptable y valiosa del sistema aeronáutico, pero es también la más vulnerable a influencias que pueden afectar negativamente su comportamiento" (OACI, 1998, 1-1-13). Se ha tenido en cuenta que la actuación humana, es decir, el comportamiento de un ser humano al interior del contexto aeronáutico debe ser intervenido, medido y controlado, de tal manera que se fomente y se acoja a los estándares de seguridad, necesarios para evitar incidentes y/o accidentes.

De ahí entonces, puede surgir la cuestión: ¿¿de qué dependería la seguridad en el contexto aeronáutico? La seguridad, al menos en la aviación, depende no sólo de factores técnicos, referidos a la máquina, la ergonomía y la ingeniería, sino también a factores que son humanos (Alonso, 2013).

Existe evidencia de que la psicología aportó al campo aeronáutico desde la Primera Guerra Mundial. Tal como lo señala Sáiz y Sáiz (2012) entre 1887 y 1940 se da un desarrollo de la psicología del transporte. Es a partir de 1911 que aparecen los primeros estudios de la psicología enfocada en la aviación. En Italia, Luigi Falchi realiza el primer perfil psicológico de un piloto, en el cual se tuvieron en cuenta aspectos como la reacción ante diferentes estímulos, así como evaluar los niveles de atención, generando una primera caracterización de los pilotos. Desde 1912, hasta aproximadamente 1919, se da el primer desarrollo de la psicología de la aviación. Durante este primer lapso el psicólogo aeronáutico, en sus comienzos, centró su atención en:

1) Detectar la aptitud para captar rápidamente acontecimientos y reaccionar ante los mismos de manera adecuada.

Numeración de página Manual de Instrucción en Factores Humanos de la Organización Internacional de la Aviación Civil, OACI. 
2) Identificar la capacidad de atención ya concentrada y distribuida. Es decir, la capacidad para focalizar la atención sobre uno y varios elementos simultáneamente.

3) Identificar la capacidad de observación precisa y rápida frente a la información que se le proporciona.

4) Determinar cuáles son los niveles de emotividad de los tripulantes que se evaluaban en el contexto de la guerra.

Para lo anterior, y en el contexto de la Primera Guerra Mundial, se encuentran trabajos sobre la velocidad para la reacción, las reacciones emotivas y la capacidad para tener un control inhibitorio de las reacciones emocionales (Sáiz y Sáiz, 2012). Así mismo, se comienzan a diseñar los test y las herramientas para evaluar y medir: la estabilidad emocional, percepción de la inclinación, oscilación, reacción visual, reacción de equilibrio, diferencia de equilibrio, alerta mental (por ejemplo, la prueba de Alerta Mental de Thorndike), interés y ejecución atlética.

Hein (2010) afirma que respecto a la psicología que interviene en el contexto de la aviación, hay exigencias que se formulan al personal inmerso en la operación aérea. Estas exigencias pueden considerarse paradójicas, según este mismo autor, pues apuntan a que la actuación humana se perfile en pro de la seguridad y se enfoquen en modificar el comportamiento. Estas exigencias son:

1) Someterse a las regulaciones, reglas, procedimientos frente a la necesidad de ser líderes con iniciativa y responsabilidad ante las situaciones de emergencia. Lo que contrasta además con los requisitos de destacarse con cierto nivel de mando siendo subordinados por los procedimientos.

2) La calma y el control emocional necesario para enfrentar situaciones de emergencia, y la necesidad de ser impulsivos y reaccionar en márgenes de tiempo reducido, buscando respuestas adaptativas a los diversos acontecimientos que se pueden presentar.

3) Alternar las horas de trabajo con largas jornadas de descanso. Que implica tolerar situaciones de estrés y la fatiga de vuelo. Es decir, tolerar reacciones 
fisiológicas asociadas al desgaste que genera el vuelo y los turnos de trabajo. Lo cual tiene en cuenta que la fatiga es un:

Estado fisiológico que se caracteriza por una reducción de la capacidad de desempeño mental o físico debido a la falta de sueño o a períodos prolongados de vigilia, fase circadiana, o volumen de trabajo (actividad mental y/o física) y que puede menoscabar el estado de alerta de un miembro de la tripulación y su habilidad para operar con seguridad una aeronave o realizar sus funciones relacionadas con la seguridad operacional (OACI, 2013. p. xi).

4) Tener la capacidad de tomar decisiones "rápidas y acertadas sin una información completa versus buscar siempre toda la información posible" (Hein, 2010, p. 63); es decir, alta habilidad para la toma de decisiones, identificando el tipo de situación, el tipo de riesgo o peligro, sin que necesariamente se cuente con las variables que conforman dicho evento.

5) Motivación por la actividad que realizan teniendo en cuenta los retos, los peligros y el nivel de responsabilidad que tiene su actuación.

Ahora bien, a partir de algunos de los aspectos para tener en cuenta por el psicólogo aeronáutico, ¿cómo se puede conceptualizar la psicología aeronáutica? Todos los autores consultados coinciden en que la psicología aeronáutica o de la aviación se enmarca en la selección del personal aeronáutico, de ser así, ¿qué la diferenciaría de la psicología de las organizaciones? Podría decirse que la psicología aeronáutica es:

Una especialidad de la psicología, aplicada al estudio de los procesos psicológicos de los individuos, grupos y organizaciones, que integran el Sistema Sociotécnico Aeronáutico. En su participación dentro del campo multidisciplinario de los Factores Humanos, orienta su atención en especial hacia el estudio del error humano en la búsqueda de una performance aceptablemente segura en la operación de los sistemas aeronáuticos. Los aportes teóricos y técnicos en el análisis del nivel psicológico de integración intentan comprender y predecir en especial qué comportamientos contribuyen a la óptima performance del sistema y cuáles la degradan (Alonso, 2012; 2013). 
De ahí entonces que se proponga una delimitación del campo y se plantee la acción del psicólogo aeronáutico articulada a la selección desde la óptica de los Factores Humanos, el entrenamiento, la implementación de sistemas que sirvan como defensa frente al error humano, lo cual impactará sobre la seguridad operacional y la disminución de los niveles de accidentalidad. Tal como lo afirma Alonso (2012), la acción del psicólogo en el medio aéreo:

[...] implica desarrollos de los procesos de selección, aptitud, y entrenamiento de personas y equipos de trabajo; en el diseño, organización y control de sistemas, buscando adecuada relación de las interfases de los seres humanos entre sí y en su relación con las máquinas, los procedimientos y el ambiente de las operaciones. Sobre esta base, tiene definida participación en las estrategias de prevención y de investigación de accidentes, y por lo tanto en la seguridad operacional (Alonso, 2012; 2013).

Pareciera que la labor de la psicología aeronáutica se centrase sólo en la evaluación, identificación y medición de perfil, valoración clínica y de instrucción de pilotos. Así mismo, Oliveros amplía la posición de Alonso argumentando que la psicología aeronáutica es "el estudio de los individuos comprometidos con la aviación en actividades relacionadas" (Oliveros, 2015. p. 7). Esto es consecuente con que este campo es "una rama de la psicología aplicada que se ocupa del estudio de todos los aspectos psicológicos y conductuales que intervienen en el hombre que se encuentra o actúa en el medio aeronáutico" (Mirabal, 2011, p. 1).

Estudiar los diferentes aspectos, respuestas y comportamientos de un tripulante cabina de mando (o piloto), ubica a la psicología aeronáutica más allá del campo organizacional, o de la selección y la interacción medio laboral- individuo, y propone no sólo la selección identificando comportamientos operacionalmente seguros, o la valoración clínica, en el campo de la aviación, la psicología se considera:

[...] una disciplina científica, [...] que aporta teorías, métodos y prácticas psicológicas para explicar cómo se hacen o deberían hacer las cosas en aviación. En este sentido no es única y comparte con otras disciplinas muchos de los temas que aborda (McDonald, Johnson y Fuller, 1994, p. 10). 
La fórmula entonces que relaciona el objeto con el sujeto, al menos desde la psicología aeronáutica puede ser esquematizada así: el objeto de estudio es la actuación humana de aquellas personas que están inmersas en el contexto aeronáutico y que tengan relación con la seguridad operacional. Desde esta perspectiva, lo que se denomina actuación centra la atención en el comportamiento humano que puede ser identificado desde la selección del personal y tiene en cuenta el desempeño de un sujeto al interior del medio aeronáutico. De esta manera la relación sujeto-objeto, mediada por la noción de actuación humana tiene en cuenta:

1. Los efectos psicológicos generados por cambios de turnos y/o de los vuelos transatlánticos, los cuales son vivenciados como fatiga, desincronización de los ritmos del organismo (OACI, 1998) y jetlag.

2. Los niveles de preparación que tiene un ser humano ante cualquiera de las situaciones que se pueden presentar al interior de una aeronave (despresurización, desorientación espacial, pánico, emergencias, interceptaciones ilícitas, entre otras).

3. Los niveles de respuestas y procedimientos a seguir frente a cualquiera de estas situaciones.

4. Los niveles atencionales y capacidad de procesar la información.

5. Las aptitudes intelectuales (razonamiento numérico, verbal, mecánico y lógico).

6. La habilidad para tomar decisiones y comunicarse con el personal involucrado en la operación aérea.

La historia de la psicología aeronáutica se remonta a 1912. El contexto histórico para esta ciencia entre los ańos 1900 y 1920 estuvo marcado por las demandas de la industria del transporte, que pretendía realizar selección de su personal operativo (conductores, pilotos y demás personal encargado de responder ante los crecientes medios de transporte). Tal como afirma Terrasa:

Ante la demanda de dictámenes de capacitación (selección) para casi cualquier actividad, y la no menos demandada 'orientación', la entonces nueva Psicología 
respondió con un conjunto de propuestas que definieron lo que en Europa se llamó 'Psicotecnia', 'Psicotécnica', o 'Tecnopsicología' y en EE.UU 'Psicología Aplicada’. (2005, p. 38).

La psicología fue convocada a dar respuesta sobre el ser humano a la hora de desempeñar una actividad, para el caso sobre los medios de transporte. Ya en 1906 en algunos laboratorios de psicología en Italia, Alemania y Francia, se puede observar un interés por asuntos relacionados con el vuelo, denominados aspectos psicofisiológicos, tales como: "fatiga, dolor, funcionamiento muscular, reacción nerviosa y control de la emotividad" (Terrasa, 2005, p. 40), así como "las diferencias individuales en cuanto a capacidades y disposiciones (...) y el aprendizaje de los trabajos y sus modalidades" (p. 40, citando a Fuller y cols., 1997; Shimmin y Van Strien, 1998). Si bien para esta época la psicología aeronáutica aún no se constituía, la relación de la naciente ciencia con el nuevo artefacto que constituyó la aeronave, engendraron preguntas por la relación humano-máquina que permitiría la constitución de un campo que indagará por las respuestas de un individuo ante situaciones puntuales durante el vuelo.

Es en 1912 que se realiza la primera delimitación del campo. Se comienzan a realizar estudios de las cualidades psicofísicas, siendo éstas lo que se constituiría el primer objeto de estudio. En Italia, Agostino Gemelli, realizará la elaboración de los primeros exámenes psicológicos de aptitudes mentales para la exploración de aviadores. Este interés se formalizará aún más cuando Hugo Münsterberg plantea que: "corresponde a los psicólogos, mediante los métodos que ellos emplean, determinar las cualidades que exige una profesión dada y luego investigar si el individuo que quiere abrazar esa profesión posee verdaderamente dichas cualidades" (Terrasa, 2005, p. 40, citando a Münsterberg, 1912), ubicando la relación de la psicología como ciencia que puede estudiar al hombre en diversos escenarios de actuación. Es en 1912 que se comienza un movimiento de estudio sobre los factores psicofísicos que intervienen durante el vuelo.

La historia de la psicología aeronáutica se podría entonces esquematizar en cuatro períodos de tiempo en los cuales participaron diversos países y en los cuales la evolución de este campo es notable. A continuación, se presenta el esquema de organización histórica de la psicología aeronáutica. 
Tabla 1. Desarrollo histórico de la Psicología Aeronáutica

\begin{tabular}{|c|c|c|c|c|}
\hline Período & País & Hechos relevantes & $\begin{array}{c}\text { Concepción } \\
\text { objeto de la } \\
\text { psicología } \\
\text { aeronáutica }\end{array}$ & $\begin{array}{c}\text { Concepción } \\
\text { sujeto de la } \\
\text { psicología } \\
\text { aeronáutica }\end{array}$ \\
\hline $\begin{array}{l}\text { Primero } \\
(1912-1919)\end{array}$ & $\begin{array}{l}\text { Italia, } \\
\text { Francia, } \\
\text { Alemania, } \\
\text { Inglaterra }\end{array}$ & $\begin{array}{l}\text { - Se aplicaron los conocimientos psicológicos a la } \\
\text { selección de aviadores (se realizaba la selección y } \\
\text { clasificación de personal). } \\
\text { - Se da una constante publicación de los primeros } \\
\text { estudios psicológicos a pilotos (la publicación } \\
\text { de los trabajos de J. Camus y H. Nepper están } \\
\text { situados entre } 1916 \text { y 1919). } \\
\text { - Aparecen los primeros estudios sobre las } \\
\text { medidas de las reacciones psicomotrices de los } \\
\text { candidatos a la aviación, tiempos de reacción } \\
\text { psicomotrices, la aptitud para la aviación y } \\
\text { reacciones emotivas de los candidatos a la } \\
\text { aviación. }\end{array}$ & $\begin{array}{l}\text { Variables } \\
\text { psicofisiológicas: } \\
\text { tiempo de } \\
\text { reacción, niveles } \\
\text { de atención, } \\
\text { respuestas } \\
\text { emotivas }\end{array}$ & Piloto \\
\hline $\begin{array}{c}\text { Segundo } \\
(1920-1945)\end{array}$ & $\begin{array}{l}\text { Italia, } \\
\text { Francia, } \\
\text { Alemania, } \\
\text { Estados } \\
\text { Unidos, } \\
\text { Inglaterra }\end{array}$ & $\begin{array}{l}\text { La finalización de la Primera Guerra Mundial } \\
\text { y los comienzos y finalización de la Segunda } \\
\text { Guerra Mundial, plantean la necesidad de } \\
\text { identificar qué tan preparado está un piloto para } \\
\text { volar. } \\
\text { - Se realizan las primeras investigaciones que } \\
\text { indagan por la incidencia humana en la } \\
\text { accidentalidad aérea. En estos se encuentra que } \\
\text { los factores psíquicos aparecían como principales } \\
\text { agentes causales en las caídas de los aviones. } \\
\text { - Se publican } 271 \text { trabajos en torno a la psicología } \\
\text { en relación con la aviación (Sáiz. y Sáiz, 2012) } \\
\text { clasificados en los siguientes grupos temáticos: } \\
\text { 1. Búsqueda de aparatos, test y métodos para la } \\
\text { medida de aptitudes dirigidos a la selección. } \\
\text { 2. Análisis de los factores humanos que se ven } \\
\text { alterados durante el vuelo. } \\
\text { 3. Estudio de las aptitudes que debe tener un } \\
\text { aviador. } \\
\text { 4. Propuestas para la selección de aviadores. } \\
\text { 5. Propuestas de medición de las condiciones del } \\
\text { aviador antes del vuelo. } \\
\text { 6. Alteraciones que se producen en los aviadores } \\
\text { como consecuencia de haber volado. } \\
\text { 7. Estudios perceptuales o sensoriales y motrices. } \\
\text { 8. Visiones generales o recopilaciones. }\end{array}$ & $\begin{array}{l}\text { Aptitud para el } \\
\text { vuelo }\end{array}$ & $\begin{array}{c}\text { Pilotos y } \\
\text { observadores de } \\
\text { vuelo. }\end{array}$ \\
\hline
\end{tabular}




\begin{tabular}{|c|c|c|c|c|}
\hline $\begin{array}{c}\text { Tercero } \\
(1946-1973)\end{array}$ & $\begin{array}{l}\text { Estados } \\
\text { Unidos }\end{array}$ & $\begin{array}{l}\text { - La posguerra deja avances en la psicología } \\
\text { clínica que permiten identificar situaciones } \\
\text { patológicas en pilotos que experimentaron la } \\
\text { guerra. } \\
\text { - Se empiezan a establecer los primeros } \\
\text { profesiogramas de pilotos, así como el } \\
\text { entrenamiento y especialización en la } \\
\text { tarea. Los test psicométricos comienzan a } \\
\text { ser instrumentos de valoración de rasgos } \\
\text { caracterológicos y los procesos sensorios, } \\
\text { perceptuales y de reacción. } \\
\text { Aparece la preocupación por la psicopatología } \\
\text { del piloto, se empiezan a evaluar fobias, } \\
\text { psicosis, filias. } \\
\text { - Se fundan los primeros laboratorios de } \\
\text { psicología en aviación. } \\
\text { Estados Unidos adquiere relevancia en los } \\
\text { estudios del comportamiento del ser humano } \\
\text { en el contexto aeronáutico. } \\
\text { - Se crean las sociedades de Factores Humanos, } \\
\text { las cuales convocan psicólogos que } \\
\text { adelanten estudios sobre la influencia del } \\
\text { comportamiento en la seguridad aeronáutica. } \\
\text { - Aparece el Modelo SHELL el cual es } \\
\text { elaborado por Elwyn Edwards (1972) el cual } \\
\text { conceptualiza el ser humano (Liveware) en } \\
\text { interacción con los diferentes componentes del } \\
\text { sistema aeronáutico (ambiente-Enviroment, } \\
\text { Máquina-Hardware, Soporte Lógico- } \\
\text { Software). } \\
\text { entrenamiento de pilotos en simuladores } \\
\text { de vuelo. En estos se buscó aproximar la } \\
\text { psicología aeronáutica a estudiantes no } \\
\text { procedentes del mundo militar. } \\
\text { se estructura con un carácter divisional y } \\
\text { propone: "la psicología como una ciencia, } \\
\text { pomo una profesión, y como medio para } \\
\text { 2005). }\end{array}$ & $\begin{array}{l}\text { Factor Humano } \\
\text { en la Aviación } \\
\text { (entendido } \\
\text { como la } \\
\text { actuación } \\
\text { humana) }\end{array}$ & $\begin{array}{c}\text { Pilotos y } \\
\text { observadores de } \\
\text { vuelo. }\end{array}$ \\
\hline $\begin{array}{c}\text { Cuarto } \\
\text { (1974- } \\
\text { Actualidad) }\end{array}$ & $\begin{array}{l}\text { Estados } \\
\text { Unidos, } \\
\text { España, } \\
\text { Argentina }\end{array}$ & $\begin{array}{l}\text { - Aparece el CRM (Copkit Resources } \\
\text { Management - Administración de Recurso } \\
\text { en Cabina), el cual buscaba aumentar el } \\
\text { desempeño de la tripulación y disminuir los } \\
\text { accidentes a partir de la comunicación, toma } \\
\text { de decisiones y trabajo en equipo. } \\
\\
\text { - La psicología aeronáutica interviene en la } \\
\text { capacitación para la prevención de accidentes y } \\
\text { la toma de conciencia de riesgos generados por } \\
\text { factor humano. }\end{array}$ & & $\begin{array}{l}\text { Personal } \\
\text { involucrado con } \\
\text { la seguridad } \\
\text { operacional } \\
\text { (tripulantes } \\
\text { cabina de } \\
\text { pasajeros, pilotos. } \\
\text { Luego aparecerá } \\
\text { la gestión de } \\
\text { recursos de } \\
\text { mantenimiento }\end{array}$ \\
\hline
\end{tabular}




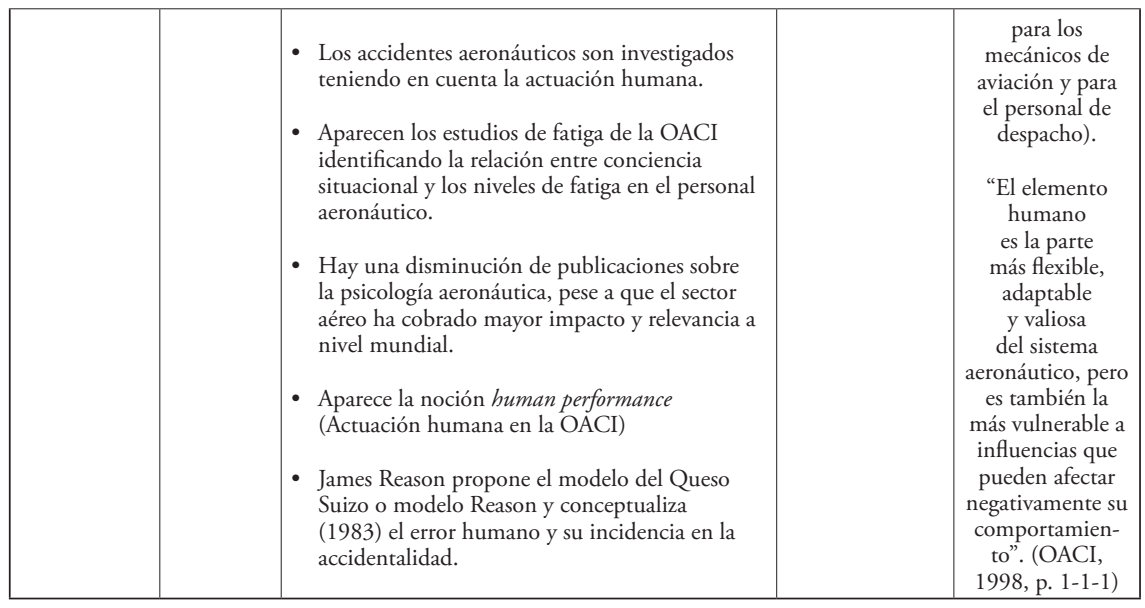

Nota: Elaboración propia.

El desarrollo de la psicología de la aviación entre 1920 y 1945 estuvo marcado por la Primera y segunda Guerra Mundial, lo que permitió la realización de preguntas sobre el ser humano a nivel de su comportamiento y sus reacciones en el contexto del vuelo. En la posguerra de la Segunda Guerra Mundial, aparece, por un lado, la necesidad de identificar si hay psicopatología en los pilotos y por otro lado, emergen estudios sobre el entrenamiento y la selección, no sólo referidos a la aviación militar sino también a la aviación civil.

No obstante, desde la aparición de los primeros estudios de la psicología en la aviación en 1912, hasta la actualidad, el objeto de la psicología en el campo aeronáutico pareciera difuso, dado que no ha existido una agrupación de conceptos que definiera un objeto único. Sin embargo, la caracterización de cualidades psicofísicas entre las que se tenía en cuenta los tiempos de reacción, los niveles de emotividad, la capacidad para concentrar y distribuir la atención, la precisión y la agilidad, han permanecido como constantes de estudio que caracterizan al menos lo que el psicólogo aeronáutico ha investigado, intervenido y tratado de enmarcar en un marco conceptual. De esta forma, se puede identificar de qué manera el objeto de estudio de los psicólogos aeronáuticos fueron las características psicofísicas de un piloto durante el vuelo, teniendo 
en cuenta que estas cualidades estaban definidas siempre por las respuestas de un individuo que desempeña un rol en el medio aeronáutico.

Es con el final de la Segunda Guerra Mundial, con los aportes y avances en cuestiones de psicometría y las consideraciones psicopatológicas que se tenían para aquel entonces, que se modifica la conceptualización del objeto, el cual fue delimitado como el comportamiento del aviador (piloto) y del observador de vuelo en las condiciones propias de un vuelo. Lo anterior permitió que tras la guerra las cualidades psicofisiológicas fueran conceptualizadas como factores humanos en aviación, los cuales son efecto de las guerras en tanto que "se extendió la capacidad humana a su máximo mediante la aplicación del conocimiento de los factores humanos, a veces con riesgo de ignorar las limitaciones humanas" (OACI, 1998, p. 1-1-1).

Luego del accidente de los Rodeos ocurrido en Tenerife el 22 de marzo de 1977, en el que dos Boeing 747 colisionan, y que ocasiona 583 muertes, la investigación de los accidentes aéreos cambia, pues comienza a tenerse en cuenta la relación de la actuación humana en los incidentes y accidentes aeronáuticos. Luego de este acontecimiento, se identifica que las causas de este evento estuvieron relacionados con "una falla en los procedimientos normales de comunicación y la mala interpretación de mensajes verbales" (OACI, 1998. p. 1-1-2). Lo anterior, hace emerger la necesidad de conceptualizar el 'error humano', las causas de este y los efectos a nivel de seguridad operacional como parte del repertorio y campo de actuación de la psicología aeronáutica.

Es con James Reason (1990) que el error humano se conceptualiza. Los factores humanos entonces dan lugar a pensar en las capacidades del ser humano y las limitaciones que tienen frente a las demandas del medio aeronáutico, estructurando el objeto de estudio de la psicología aeronáutica como la 'actuación humana' la cual comprende las capacidades de un individuo que le posibilitan ejercer en el sector aéreo teniendo en cuenta el procesamiento o tratamiento de la información y las habilidades no técnicas (Flin., O’Connor., \& Crichton, 2008) y los procesos psicológicos que engendran el error, así como a las limitaciones como la fatiga, el estrés, alteraciones del sueño, fallos y limitaciones fisiológicas derivadas de las condiciones del vuelo. 


\section{Psicología Aeronáutica y Psicopatología}

El desarrollo de los medios de transporte, las migraciones y los cambios sociales que se dan entre finales del siglo XIX y comienzos del XX, generan preguntas por lo humano que convocaron a la psicología. El sector aeronáutico la interrogan también, ya los hermanos Wright, primeros hombres en sostener un vuelo propulsado controlado (Alonso, 2001), pactan cuatro años después de su primer vuelo (1907), el uso de aeroplanos para el ejército norteamericano asesorando a las fuerzas militares, acción que en el período en que se dio la Primera Guerra Mundial llevó a las primeras aproximaciones científicas de la psicología sobre el personal aeronáutico.

El antecedente de la Primera y Segunda Guerra Mundial, permitieron desarrollos tecnológicos que consolidaron los planteamientos de Leonardo Da Vinci sobre los aeromodelos (Alonso, 2001). El período que comprende la Primera Guerra Mundial plantea preguntas sobre la selección de pilotos tales como: “`ser valiente es suficiente para pilotar? o si se quiere, ¿esta característica es la única que debe estar presente para seleccionar un buen piloto, o son necesarias otras aptitudes psicofisiológicas?” (Sáiz y Sáiz, 2012, p. 11).

La Unidad Administrativa Especial de la Aeronáutica Civil Colombiana (UAEAC) considera que la psicología Aeronáutica es la "rama de la psicología que se ocupa del estudio de todos los aspectos psicológicos y conductuales que intervienen en el personal que se encuentra o actúa en el medio aeronáutico" (RAC 67, 2015, p. 3). A su vez Jensen (1997, citado en Alonso, 2011), establece que la psicología aeronáutica es "una aplicación de la psicología al campo del desarrollo y la operación de sistemas aeronáuticos seguros y efectivos, desde el punto de partida del operador humano" (p. 9).

La primera mitad del siglo XX es la oportunidad para que la psicología se acercara al medio de la aviación con el fin de entender al piloto, es decir, para interpretar los factores psicológicos implicados en un tripulante. Los estudios sobre las respuestas a estímulos, la fatiga mental, desnutrición, cefalalgia, estados de paroxismo, fobias y neurosis (Bandrés y Llavona, 1996) que se suman a la pregunta por las aptitudes psicofisiológicas.

A partir de la segunda mitad del siglo XX, la psicología es convocada para 
realizar no sólo procesos de selección sino también de intervención, siendo ella una de las ciencias que aportan al entendimiento de los Factores Humanos. Durante este período, la psicología aeronáutica abarca no sólo al tripulante, sino a las diferentes personas que intervienen en la operación aérea. Se redefine el campo de intervención, y el psicólogo aeronáutico entonces puede encontrar su hacer en la evaluación, el diagnóstico y la intervención.

Esta clasificación abarca las diferentes actividades propuestas por Alonso (2012), en las que se pueden encontrar que la evaluación comprende: identificación de aspectos normales y anormales, rasgos de personalidad, diseño y aplicación de baterías de pruebas; así mismo, los Reglamentos Aeronáuticos Colombianos -RAC- $(67,2015)$ establecen la evaluación de aptitudes intelectuales, estructura de personalidad, motivación por el medio aeronáutico y habilidades específicas, tolerancia a la presión y al estrés. Pareciera entonces haber un consenso sobre el campo de ocupación de un psicólogo al interior del medio aeronáutico, a su vez, pareciera existir una noción clara de lo que la actuación y las limitaciones del ser humano son, no obstante, ¿puede existir claridad sobre lo que el psicólogo en el campo aeronáutico define como salud mental?

Para dar una respuesta, es necesario articular al sujeto del que se habla en el medio aeronáutico; es decir, de una persona cuyas labores están involucradas con la seguridad operacional. Una de las miradas que puede permitir esta comprensión es la del transdiagnóstico, se podría hacer una aproximación, en la cual se articule el individuo y sus motivaciones, a la luz de una mirada en la cual su contexto, puede generar determinaciones sobre su actuar. No obstante, no podría pensarse sólo en ese eje, pues, ¿cómo articular las decisiones personales y subjetivas sólo a una determinación contextual?, es más, desde el caso del primer oficial de Germanwings, más allá de la posible depresión, ¿hablamos de un suicidio o de un homicidio?

Es necesario hacer un esfuerzo por identificar los aspectos no sólo comportamentales, sino motivacionales en el contexto inmediato, es decir, en la cual el elemento humano, el piloto, el vuelo, su aparente condición de salud no supervisada ni por la autoridad aeronáutica ni por la aerolínea, esbozan más preguntas; estas son tanto de orden ético, como político y de políticas, en las 
cuales la pregunta por lo normal y salud mental, insertan al psicólogo en un rol crítico frente a la situación planteada.

Desde la evaluación y diagnóstico, en el cual se pretende identificar las características y estructura de personalidad, obliga tener en cuenta las nociones y conceptos de la psicología alrededor de la personalidad. No obstante, en el momento de evaluar, ¿se tienen en cuenta los conflictos subjetivos, el contexto en el cual se desarrolla a personalidad y en los cuales el contexto mismo ofrece variables de análisis para llegar a una comprensión de los que puede entenderse como personalidad?

Si se tuviera en cuenta esto, por ejemplo, al momento de hacer evaluaciones de tripulantes, se tendría una visión más enriquecedora en la selección y en el entendimiento de la limitación del ser humano en el contexto del vuelo, a saber, que el elemento humano no está diseñado fisiológicamente para volar, pero, aun así, la aeronave es un medio de transporte, que goza de prestigio social en el cual la seguridad aérea es el objetivo siempre. Paradójicamente, esta seguridad oscila en las demandas hacia el tripulante en las cuales se distinguen responsabilidades, pero se exige a su vez que tenga una personalidad apta para el vuelo. Entre estas exigencias, siempre paradójicas, se encuentran, tal como lo afirma Lotero Serrano (2005):

- Someterse a todas las regulaciones, procedimientos y reglas versus tener iniciativa y responsabilidad en la ejecución de la tarea.

- Control de sí mismos, bajos niveles de impulsividad versus capacidad de reaccionar de manera ágil y adaptativa a las situaciones que se presentan.

- Alternar los tiempos de trabajo con tiempo proporcional de descanso.

- Tomar decisiones de manera rápida y acertada, sin tener la información completa, versus buscar siempre toda la información posible sobre sus funciones.

- Alta motivación y afecto positivo por lo que hacen versus respeto por el peligro propio de sus funciones.

- Tener confianza en sí mismo versus no tener sentimientos de grandiosidad.

- Ser líderes y ser capaces de subordinarse con facilidad. 
¿Cómo articular estas exigencias a una reflexión sobre lo psíquico? ¿Cómo entender la coherencia en el momento de evaluar un tripulante en relación con las demandas paradójicas propias del medio aeronáutico? ¿Se requiere un perfil psicopatológico para identificar si quien desea ser tripulante se adapta a estas exigencias? Se podría pensar incluso una falta de coherencia en las preguntas anteriores. No obstante, ilustra de qué manera lo que se denomina perfil, está suscrito a un contexto y si bien estas demandas son propias del mismo, ¿sería apto por ejemplo un médico, un político, un psicólogo para este perfil de tripulante? Precisamente se puede evidenciar en este punto, que la relación contextual, permite lecturas que trascienden el diagnóstico e ilustran dos elementos adicionales: una evaluación más amplia y una intervención del psicólogo enfocada en las necesidades propias del campo, así como focalizadas en las demandas propias del medio aeronáutico.

La intervención en este campo consta de realizar acciones de orientación y asesoramiento psicológico tendientes a la promoción de la salud y la prevención de sus alteraciones; construir y desarrollar métodos, técnicas e instrumentos psicológicos estudiar, orientar y esclarecer los conflictos interpersonales e intergrupales en el contexto de la estructura y dinámica de las instituciones para prevenir accidentes. Desde esta perspectiva, ¿cómo optimizar los niveles de seguridad operacional y aérea desde una mirada psicopatológica que involucre una mirada contextual?

La evaluación de la aptitud psicofísica, tal como se encuentra denominada por los Reglamentos Aeronáuticos Colombianos - RAC - y por los Reglamentos Aeronáuticos Latinoamericanos — LAR — de tripulantes de vuelo (Tripulantes Cabina de Mando — pilotos_y Tripulantes Cabina de Pasajeros —auxiliares de vuelo-), precisan de que todo personal evaluado y declarado psicológicamente apto, sea poseedor de salud mental. Teniendo en cuenta las demandas propias del medio aeronáutico, ¿cómo evaluar la salud mental?

El período entreguerras, así como el período previo y posterior a la Segunda Guerra Mundial, y apoyándose en las baterías psicométricas, así como en los test proyectivos, surge una preocupación por el asunto de lo psicopatológico en el sector aeronáutico. Esta preocupación se focalizó en identificar manifestaciones psicopatológicas en dos niveles; el primero el que podemos 
denominar "aptitudes intelectuales" (UAEAC, 2015, p. 27), y el segundo, en el que se trató de identificar si la persona evaluada y que posteriormente desempeñaría tareas relacionadas a la seguridad, no poseía indicadores de patología psicológica.

La salud mental en este campo adquiere relevancia con las primeras investigaciones realizadas en 1919 cuando Otto Selz, Wilhelm Benary, E. Stern y Arthur Kronfeld publican el libro "Estudios sobre la aptitud psicológica para la actividad de vuelo" (Citado por Sáiz y Sáiz, 2012, p. 22) producción del análisis de Selz sobre los accidentes aéreos. Selz encontró que el 53\% de los accidentes de aviación se deben al factor humano individual refiriéndose a las aptitudes del piloto. Destaca que el $40 \%$ de los accidentes ocurrían durante el aterrizaje, seguida por el despegue. Dado lo anterior, propone un examen en el que se simularan las dificultades que presentan las maniobras de despegue y aterrizaje.

El examen se enfocó en la distribución de la atención, la tendencia a distraerse del piloto, la velocidad de abstraer información y reaccionar teniendo en cuenta la exactitud y precisión, medición de la fatiga, el equilibrio emocional, la coordinación, la percepción de la posición corporal y el sentido de la orientación.

Así mismo, como lo señala Terrasa (2102), en países como Italia y Francia, la publicación de los trabajos de J. Camus y H. Nepper están situados entre 1916 y 1919, en los cuales se propendía por una medición de las reacciones psicomotrices de los candidatos a la aviación, tiempos de reacción psicomotrices de los aspirantes a pilotos, aptitud para la desempeńar funciones en el medio aéreo o estudios sobre las reacciones psicomotrices y de las reacciones emotivas de las personas evaluadas, ven la luz en esa época difundiendo el proceso seguido para la determinación de buenos aviadores, método que sería introducido en Inglaterra por Graeme Anderson en 1917 y en Alemania por Gustav Braunbeck y Walther Isendahl en 1916 y 1919.

Los diferentes estudios apuntaron al establecimiento de medidas que permitieran cierta normalización de estándares relacionados al vuelo. No obstante, sólo es hasta la década de los años cincuenta del siglo XX que se establecen estas mediciones en relación con la psicopatología en indagaciones particular- 
mente asociadas a psicosis, manías, fobias, depresiones y estrés. Así mismo, el estudio de otros factores asociados a vuelo, como efecto de la guerra, se centra el estudio en: la fatiga, el estrés asociado a la guerra, la personalidad del piloto (rasgos de personalidad, respuestas situacionales y respuestas emocionales), estabilidad emocional y emotividad, niveles de extraversión, niveles de agresividad, filias, fobias, psicosis. Se comienza a evaluar a nivel aptitudinal en el cual se indaga por las habilidades espaciales y la psicomotricidad.

A partir de los estudios y mediciones realizadas se comienzan a establecer normativas que en lo relacionado a la aviación, revelan factores discriminativos en los perfiles de las personas involucradas con el medio aeronáutico y cuyas tareas están asociadas con la seguridad Operacional. En el Capítulo 67 de los Reglamentos Aeronáuticos de Colombia (RAC), Normas para el Otorgamiento del Certificado Médico (UAEAC, 2015), se puede hallar que es un factor excluyente de actividades de vuelo, aquellas que provoquen o empeoren problemas o desórdenes "de carácter ocupacional, social, mental o físico" (UAEAC, 2015, p. 7). Para lo anterior, en Colombia, se emplea el marco de los Trastornos de la salud mental codificados en la Clasificación Internacional de Enfermedades de la Organización Mundial de la Salud.

Se plantean entonces requisitos de orden psicofísicos que se pueden agrupar en tres indicadores:

1. Quien tenga un diagnóstico de depresión y tenga consumo de medicamentos que traten esta enfermedad (antidepresivos)

\footnotetext{
“[...] debería considerarse psicofísicamente no apto. Sin embargo, si la patología o su tratamiento farmacológico ha sido objeto de investigación, de acuerdo a las mejores prácticas médicas y se ha estimado que es improbable que comprometa la seguridad operacional, será la Junta Médica, el organismo calificado para otorgar o rechazar la dispensa médica”. (UAEAC, 2015).
}

2. El personal aeronáutico, deberá en todo momento estar dispuesto a mostrar que no consume sustancias psicoactivas.

3. No debe haber presencia de historial clínico de:

- Trastorno mental orgánico. 
- Trastorno mental o del comportamiento debido al uso de sustancias psicoactivas; (éstos incluyen el síndrome de dependencia inducida por la ingestión de bebidas alcohólicas u otras sustancias psicoactivas).

- Esquizofrenia o un trastorno esquizotípico o delirante.

- Trastorno del humor (afectivo).

- Trastorno neurótico, relacionado con el estrés o somatoforme.

- Síndrome de comportamiento relacionado con perturbaciones psicológicas o factores físicos.

- Trastorno de la personalidad o del comportamiento adulto, particularmente si se manifiesta a través de actos manifiestos repetidos.

- Retardo mental (discapacidad).

- Trastorno del desarrollo psicológico.

- Trastorno del comportamiento o emocional, con aparición en la infancia o en la adolescencia.

- Trastorno mental que no se ha especificado de otra manera, que conforme a las mejores prácticas de la psiquiatría (apoyada por la psicología clínica), implique riesgo y pueda impedirle ejercer con seguridad las atribuciones correspondientes a la licencia que solicita o ya posee. (UAEAC, 2015).

La psicología aeronáutica entonces, se articula a la psicopatología de manera estrecha, trata de identificar situaciones que puedan afectar la seguridad y generar impases en lo concerniente a las respuestas que puede dar el sujeto en contra de los parámetros establecidos para conservar un estado de seguridad y de minimizar los riesgos. Uno de los casos que se pueden encontrar sobre este aspecto, ocurrió en 2015 con la aerolínea GermanWings.

El 24 de marzo de 2015, el caso del piloto de la aerolínea GermanWings, generó a nivel mundial cuestionamientos sobre la salud mental. El primer oficial, Andreas Günter Lubitz, luego que el comandante se ausentara de la cabina de mando, se encierra en la misma y se estrella en la región Provenza-Alpes-Costa Azul. Las discusiones de los medios de comunicación al 
respecto formulan todo tipo de hipótesis, una de ellas, sobre la certificación aeromédica del tripulante, en la que se afirma que tenía antecedentes de depresión y tendencias suicidas. Frente a esas afirmaciones no hay datos claros, y no es allí donde reside el problema por el cual la psicopatología se tendría que preguntar, es decir, no se trata de una posibilidad de identificar que por un diagnóstico de depresión no se acaba con la vida de 150 personas. Este hecho coincide con que por lo menos en Colombia, en 2015, se publique el Capítulo 67 de los Reglamentos Aeronáuticos Colombianos, pues ineludiblemente es tarea del psicólogo aeronáutico indagar por antecedentes psicopatológicos en el personal aeronáutico.

\section{Psicología Aeronáutica en el contexto colombiano}

En el desarrollo de la psicología aeronáutica se puede encontrar que desde sus comienzos hubo un interés investigativo por el ser humano en relación con su desempeño en condiciones previas y durante el vuelo. Se puede apreciar que durante los primeros años en los que se desarrolló la psicología aeronáutica, se puede evidenciar un creciente interés por articular la actividad aérea a la psicología. Sin embargo, a medida que se fue consolidando, la actividad y producción académica e investigativa fue disminuyendo. Es a partir de los años 50 del siglo XX que se consolidan los primeros laboratorios de psicología aeronáutica, que la actividad productiva disminuye y se centra más en la experimentación, de lo cual no se pueden recuperar informes de investigación o ensayos. Es a partir de la década de 1980 que se encuentran algunos informes y textos sobre la psicología aeronáutica.

En Colombia, la autoridad aeronáutica establece que la salud mental debe ser evaluada, y se deben descartar los trastornos mentales. Esta es una política que se acoge a lo establecido por la Organización Internacional de la Aviación Civil (OACI), es decir, que opera a nivel mundial, o al menos para aquellos países que se acojan a lo estipulado por esta organización. Lo anterior supone una noción de salud mental, estandarizada para todos los países que participan de la OACI, Colombia entre ellos. Esto implica una regla general sobre lo que es salud mental. 
La investigación de accidentes aéreos en Colombia en 1975 dio origen a un estudio que terminó en la implementación de un programa pionero en 1979 el cual buscaba orientar y supervisar la selección de pilotos y realizar seguimiento de los pilotos que estaban en formación. En 1993 con el Decreto 2794 del 31 de diciembre, se creó la División de Supervisión de Aptitud, la cual en julio de 1996 por la Resolución 04555 se llamaría luego División de Medicina Aeronáutica, que se convertiría en la Dirección de Medicina de Aviación en 2004. Esta situación cambia en 2017, y se convierte en el Grupo de Medicina de Aviación y Certificación Aeromédica.

Desde la creación de un ente en Colombia que vigile y supervise la labor de la psicología en lo que concierne al medio aeronáutico, se han realizado capacitaciones y seminarios de formación académica, entre los cuales se pueden encontrar:

- Primer Ciclo de Conferencias Sobre Psicología Aeronáutica.

- Seminario Sobre Síndrome de Estrés postraumático en el Ámbito Aeronáutico, por Interferencia ilícita (secuestro).

- Seminario de Manejo de Crisis por secuestro.

- Implementación de las LAR (Reglamentos Aeronáuticos Latinoamericanos) 67.

- Participación en el Desarrollo e implementación del programa de alcohol y drogas a nivel Nacional.

- Participación en investigación de accidentes desde el punto de vista de Factores Humanos.

- Necropsia psicológica en casos de accidentes fatales de aviación.

Así mismo, se identifica que la producción académica en Colombia sobre el tema es baja y que a nivel mundial, esta producción disminuyó con relación a la segunda década del siglo XX.

En Colombia la cantidad de psicólogos que se encuentran en el campo aeronáutico es reducida y las bases de datos que se tienen por parte de la Autoridad Aeronáutica (UAEAC) contienen pocos profesionales que se des- 
empeñan en el medio, y la misma, aún se encuentra en construcción. Además, se ha establecido que, si un psicólogo desea intervenir en el sector aéreo, es necesario que tenga aval por parte de la Dirección de Medicina de Aviación. Para los Centros de Instrucción, por ejemplo, hay regulaciones específicas tal cual se establece en los Reglamentos Aeronáuticos Colombianos, RAC 67, en el cual se puede hallar:

[...] debe tener conocimientos y experiencia en el entorno aeronáutico de por lo menos dos (2) años y, para adelantar las respectivas evaluaciones, requerirá autorización específica del área de medicina aeronáutica de la UAEAC, una vez haya recibido la inducción impartida por los profesionales de dicha área y acreditada su idoneidad (UAEAC, 2015).

Lo anterior reduce la posibilidad de ser reconocido como psicólogo aeronáutico, reforzado esto porque en ninguna de las universidades que se ofrecen programas de psicología en Colombia, se ofrece formación académica en relación con este campo. Los psicólogos que se encuentran en la base de datos de la autoridad aeronáutica fueron consultados con el fin de identificar de qué manera conciben el objeto, las funciones y las limitaciones del ejercicio del psicólogo aeronáutico en Colombia.

Se encuentra que la actuación humana, la estructura de personalidad, las manifestaciones psicopatológicas, los factores humanos en aviación y la aptitud psicológica, son los componentes del objeto de estudio de la psicología de la aviación. Lo anterior especifica las necesidades de profundizar en este campo, pues el crecimiento del sector aéreo en el mundo se ha incrementado mientras que el desarrollo de la psicología aeronáutica, por lo menos en Colombia apenas comienza a estructurarse como campo.

La sigla NGAP que es la iniciativa The Next Generation Aviation Professionals (La Nueva Generación de Profesionales de la Aviación) indica el crecimiento proyectado de la industria, lo que genera demandas de selección y formación de personal aeronáutico lo que plantea un reto para la psicología aeronáutica a nivel mundial.

La Tabla 2 (OACI, 2010) muestra la proyección de crecimiento. Este crecimiento entonces genera cuestiones sobre la necesidad de que los psicólogos 
puedan aportar a este desarrollo siempre y cuando cumplan las condiciones de formación para ello. Esto genera interrogantes sobre el trabajo académico e investigativo en el campo de la psicología aeronáutica en el mundo, y en Colombia la necesidad de formación y de ubicación en un marco conceptual de la manera en que la ciencia de psicología puede conformar un campo que, si bien lleva consolidándose más de cien años, apenas se empieza a esbozar en nuestro país.

Tabla 2 .

Resultados del informe de la ITQI de la IATA, 2009

\begin{tabular}{l|l|l}
\hline Demanda de pilotos y capacitación & 2018 & 2026 \\
\hline Total de pilotos necesarios para pilotar aeronaves nuevas & 193100 & 350200 \\
\hline Nuevos pilotos para aeronaves adicionales & 135000 & 227500 \\
\hline $\begin{array}{l}\text { Pilotos nuevos necesarios para cubrir la diferencia entre la } \\
\text { fluctuación y la jubilación }\end{array}$ & 72600 & 125400 \\
\hline $\begin{array}{l}\text { Total de nuevos pilotos (aeronaves adicionales y fluctuación) que } \\
\text { necesitan capacitación desde el principio }\end{array}$ & 207600 & 352900 \\
\hline $\begin{array}{l}\text { Total de pilotos nuevos que necesitan capacitación de transición } \\
\text { para aeronaves de reemplazo }\end{array}$ & 57930 & 122700 \\
\hline $\begin{array}{l}\text { Demanda de mantenimiento } \\
\text { Total de mecánicos necesarios para las aeronaves adicionales }\end{array}$ & 2018100 & 2026 \\
\hline Total de mecánicos incluida la fluctuación y la jubilación & 405500 & 739000 \\
\hline
\end{tabular}

Informe de la ITQI de la IATA, 2009, sección 4.1, pags. 9-10

Nota: Recuperado de "Respuesta al llamado de la industria: Simposio NGAP 2010", OACI, 2010, Revista de la OACI, 65(5), pp. 5-16.

Hoy, el medio aeronáutico crece, el avión sigue siendo el medio de transporte más seguro que existe y la necesidad de la participación de psicólogos para que labore en este sector se hace fundamental para promover una operación segura, la cual parte de las acciones del Elemento Humano. Pese a ello, no se da formación académica sobre este campo, ni tampoco hay suficientes investigaciones que soporten la delimitación teórica del campo. Es necesario que este tema sea pensado no sólo por las universidades, sino también por los profesionales de la psicología que laboran en el medio. 


\section{Referencias}

Alonso, M. (2001). Psicología Aeronáutica. Desarrollos Actuales, Contribuciones a la Prevención de Accidentes. 28 $8^{\circ}$ Congreso Interamericano de Psicología Santiago de Chile. (Conferencia). Tomado de: http://www.modestoalonso.com. Ar/assets/2_psicologia_aeronautica.pdf.

Alonso, M. (2013). La Psicología Aeronáutica y su Contribución a la Seguridad Aeroespacial. Rev. Argentina de Psicología, Edición 52.18 páginas.

Alonso, M. (2011). Estrés y seguridad operacional en la actividad aeroespacial. En: III Congreso Internacional de Investigación y Práctica Profesional en Psicología XVIII Jornadas de Investigación Séptimo Encuentro de Investigadores en Psicología del MERCOSUR. Facultad de Psicología-Universidad de Buenos Aires.

Bandrés, J., y Llavona, R. (1996). La psicología aeronáutica militar en España: los pioneros (1911-1925). Psicothema, 8(3), 719-731.

FlightCol. (2017) Las proyecciones de Boeing prevén que habrá una alta demanda de profesionales para los próximos 20 años en el sector aeronáutico. Recuperado de http://www.flightcol.com/las-proyecciones-de-boeing-preveen-que-habra-una-alta-demanda-de-profesionales-para-los-proximos-20-anos-en-el-sector-aeronautico/

Flin, R., O,Connor, P., \& Crichton, M. (2008). Safety at the sharp end: a guide to non-technical skills. Ashgate Publishing, Ltd.

Mcdonal, J., \& Fuller, J. (1994). Aviation Psychology In Practice. Great Britain: Ed. Avebury.

OACI (2010). Respuesta al llamado de la industria: Simposio NGAP. Revista de la $O A C I, 65(5), 5-16$

OACI (2013). Fatigue Risk Management Systems Manual for Regulators.

Oliveros, P. (2015). ¿Cómo ha aportado la Psicología al Ámbito Aeronáutico? (Tesis de Grado). Universidad Militar Nueva Granada.

Organización de Aviación Civil Internacional (OACI). (2013). Doc 9966, Manual de sistemas de gestión de riesgos asociados a la fatiga para los encargados de la reglamentación. OACI. 187 p.

Organización de Aviación Civil Internacional (OACI). (1998). Manual de Instrucción Sobre Factores Humanos (Doc. 9683-AN/950).

Reason, J. (2000). Human error: models and management. Western Journal of Medicine, 172(6).

DOI: https://doi.org/10.17533/udea.rp.v11n1a09 
Reason, J. (1990). Human error. England: Cambridge university press.

Terrasa, M. (2005). Psicología aeronáutica en España. Una historia y una panorámica actual. (Doctoral dissertation, Universitat de València).

Unidad Administrativa Especial de la Aeronáutica Civil (UAEAC). (2015). Reglamentos Aeronáuticos Colombianos. RAC. Capítulo 67. 